\title{
Fermionic bright soliton in a boson-fermion mixture
}

\author{
Sadhan K. Adhikari* \\ Instituto de Física Teórica, Universidade Estadual Paulista, 01.405-900 São Paulo, São Paulo, Brazil \\ (Received 3 May 2005; revised manuscript received 28 June 2005; published 10 November 2005)
}

\begin{abstract}
We use a time-dependent dynamical mean-field-hydrodynamic model to study the formation of fermionic bright solitons in a trapped degenerate Fermi gas mixed with a Bose-Einstein condensate in a quasi-onedimensional cigar-shaped geometry. Due to a strong Pauli-blocking repulsion among spin-polarized fermions at short distances there cannot be bright fermionic solitons in the case of repulsive boson-fermion interactions. However, we demonstrate that stable bright fermionic solitons can be formed for a sufficiently attractive boson-fermion interaction in a boson-fermion mixture. We also consider the formation of fermionic solitons in the presence of a periodic axial optical-lattice potential. These solitons can be formed and studied in the laboratory with present technology.
\end{abstract}

DOI: 10.1103/PhysRevA.72.053608

PACS number(s): 03.75.Lm, 03.75.Ss

\section{INTRODUCTION}

There cannot be an effective evaporative cooling leading to a trapped quantum degenerate Fermi gas (DFG) due to a strong repulsive Pauli-blocking interaction at low temperature among spin-polarized fermions [1]. However, it has been possible to achieve a DFG by sympathetic cooling in the presence of a second boson or fermion component. Recently, there have been successful observations [1-4] and associated experimental [5-7] and theoretical [8-12] studies of mixtures of a trapped DFG and a Bose-Einstein condensate (BEC) by different experimental groups [1-4] in the following systems: ${ }^{6,7} \mathrm{Li}[3],{ }^{23} \mathrm{Na}-{ }^{6} \mathrm{Li}[4]$, and ${ }^{87} \mathrm{Rb}^{40} \mathrm{~K}$ $[5,6]$. Also, there have been studies of mixtures of twocomponent trapped DFGs in ${ }^{40} \mathrm{~K}[1]$ and ${ }^{6} \mathrm{Li}[2]$ atoms.

The formation and collapse of a DFG in a boson-fermion mixture ${ }^{87} \mathrm{Rb}^{40} \mathrm{~K}$ have been observed and studied by Modugno et al. $[5,10,12]$. Although, the fermion-fermion interaction at short distances is repulsive due to strong Pauli blocking and hence incapable of leading to collapse, a sufficiently attractive boson-fermion interaction could overcome the Pauli repulsion and could result in a collapse of a DGF. Bright solitons in a BEC are formed due to an attractive nonlinear atomic interaction. As the interaction in a pure DFG at short distances is repulsive, there cannot be bright solitons in a DFG.

In this paper we study the possibility of the formation of stable fermionic bright solitons in a mixture of a DFG with a BEC in the presence of a sufficiently attractive bosonfermion interaction which can overcome the Pauli repulsion among fermions. The formation of a fermionic soliton is related to the fact that the system can lower its energy by forming high density regions (the solitons) when the attraction between the bosons and fermions is large enough to overcome the Pauli repulsion in the DFG and any possible repulsion in the BEC. In particular we consider the formation of fermionic bright solitons, which can freely move in the

\footnotetext{
*Electronic address: adhikari@ift.unesp.br; URL: http:// www.ift.unesp.br/users/adhikari/
}

axial direction, in such a mixture for a quasi-onedimensional cigar-shaped geometry using a coupled timedependent mean-field-hydrodynamic model where the bosonic component is treated by the mean-field GrossPitaevskii equation [13] and the fermionic component is treated by a hydrodynamic model [11]. This time-dependent mean-field-hydrodynamic model was suggested recently by the present author [12] to study the collapse dynamics of fermions and is a time-dependent extension of a timeindependent model used for the stationary states by Capuzzi et al. [11].

Bright solitons are really eigenfunctions of the onedimensional nonlinear Schrödinger equation. However, the experimental realization of bright solitons in trapped attractive cigar-shaped BECs has been possible under strong transverse binding which, in the case of weak or no axial binding, simulates the ideal one-dimensional situation for the formation of bright solitons. The dimensionless nonlinear Schrödinger (NLS) equation in the attractive or self-focusing case [14]

$$
i u_{t}+u_{x x}+|u|^{2} u=0
$$

sustains the following bright soliton [14]:

$$
\begin{aligned}
u(x, t)= & \sqrt{2 B} \operatorname{sech}[\sqrt{B}(x-\delta+2 v t)] \\
& \times \exp [-i v(x-\delta)+i(B-v v) t+i \sigma]
\end{aligned}
$$

with four parameters. The parameter $B$ represents the amplitude as well as pulse width, $v$ represents velocity, the parameters $\delta$ and $\sigma$ are phase constants. The bright soliton profile is easily recognized for $v=\delta=0$ as $|u(x, t)|=\sqrt{2 B} \operatorname{sech}[x \sqrt{B}]$. There have been experimental [15] and theoretical [16] studies of the formation of bright solitons in a BEC. In view of this, here we study the possibility of the formation of a stable fermionic bright soliton in a boson-fermion mixture.

In recent times there have been routine experimental studies on the formation of BEC in the presence of a periodic axial optical-lattice potential [17]. This leads to a different condition of trapping from the harmonic trap and generates a 
BEC of distinct modulation. Hence we also consider in this paper the modulations of the fermionic bright solitons in the presence of an optical-lattice potential.

In Sec. II we present the time-dependent mean-field model consisting of a set of coupled partial differential equations involving the bosonic and fermionic probability densities. In the case of a cigar-shaped geometry with stronger radial trapping, the above model is reduced to an effective one-dimensional form appropriate for the study of bright solitons. In Sec. III we present our results for stationary axially-free fermionic bright solitons as well as those formed on an axial periodic optical-lattice potential. We also demonstrate the stability of the bright solitons after a perturbation is applied. The bright solitons are found to execute stable breathing oscillation upon perturbation. Finally, a summary of our findings is given in Sec. IV.

\section{NONLINEAR MEAN-FIELD-HYDRODYNAMIC MODEL}

The time-dependent Bose-Einstein condensate wave function $\Psi(\mathbf{r}, t)$ at position $\mathbf{r}$ and time $t$ may be described by the following mean-field nonlinear Gross-Pitaevskii equation [13]

$$
\left[-i \hbar \frac{\partial}{\partial t}-\frac{\hbar^{2} \boldsymbol{\nabla}_{\mathbf{r}}^{2}}{2 m_{B}}+V_{B}(\mathbf{r})+g_{B B} n_{B}\right] \Psi_{B}(\mathbf{r}, t)=0,
$$

with normalization $\int d \mathbf{r}\left|\Psi_{B}(\mathbf{r}, t)\right|^{2}=N_{B}$. Here $m_{B}$ is the mass and $N_{B}$ the number of bosonic atoms in the condensate, $n_{B}$ $\equiv\left|\Psi_{B}(\mathbf{r}, t)\right|^{2}$ is the boson probability density, $g_{B B}$ $=4 \pi \hbar^{2} a_{B B} / m_{B}$ the strength of interatomic interaction, with $a_{B B}$ the boson-boson scattering length. The trap potential with axial symmetry may be written as $V_{B}(\mathbf{r})=\frac{1}{2} m_{B} \omega^{2}\left(\rho^{2}\right.$ $\left.+\nu^{2} z^{2}\right)$, where $\omega$ and $\nu \omega$ are the angular frequencies in the radial $(\rho)$ and axial $(z)$ directions with $\nu$ the anisotropy parameter. The probability density of an isolated DFG in the Thomas-Fermi approximation is given by [10]

$$
n_{F}=\frac{\left[\max \left(0,\left\{\epsilon_{F}-V_{F}(\mathbf{r})\right\}\right)\right]^{3 / 2}}{A^{3 / 2}}
$$

where $A=\hbar^{2}\left(6 \pi^{2}\right)^{2 / 3} /\left(2 m_{F}\right), \epsilon_{F}$ is the Fermi energy, $m_{F}$ is the fermionic mass, and the function max denotes the larger of the arguments. The confining trap potential $V_{F}(\mathbf{r})$ $=\frac{1}{2} m_{F} \omega_{F}^{2}\left(\rho^{2}+\nu^{2} z^{2}\right)$ has axial symmetry as the bosonic potential $V_{B}(\mathbf{r})$, where $\omega_{F}$ is the radial frequency. The anisotropy parameter $\nu$ will be taken to be zero for axially free solitons in the following. The number of fermionic atoms $N_{F}$ is given by the normalization $\int d \mathbf{r} n_{F}(\mathbf{r})=N_{F}$.

We developed a set of practical time-dependent meanfield-hydrodynamic equations for the interacting bosonfermion mixture starting from the following Lagrangian density [12]:

$$
\begin{aligned}
\mathcal{L}= & \frac{i}{2} \hbar\left[\Psi_{B} \frac{\partial \Psi_{B}^{*}}{\partial t}-\Psi_{B}^{*} \frac{\partial \Psi_{B}}{\partial t}\right]+\frac{i}{2} \hbar\left[\sqrt{n_{F}} \frac{\partial \sqrt{n_{F}^{*}}}{\partial t}-\sqrt{n_{F}^{*}} \frac{\partial \sqrt{n_{F}}}{\partial t}\right] \\
& +\left(\frac{\hbar^{2}\left|\nabla_{\mathbf{r}} \Psi_{B}\right|^{2}}{2 m_{B}}+V_{B}\left|\Psi_{B}\right|^{2}+\frac{1}{2} g_{B B}\left|\Psi_{B}\right|^{4}\right) \\
& +\left(\frac{\hbar^{2}\left|\nabla_{\mathbf{r}} \sqrt{n_{F}}\right|^{2}}{6 m_{F}}+V_{F}\left|n_{F}\right|+\frac{3}{5} A\left|n_{F}\right|^{5 / 3}\right)+g_{B F} n_{F}\left|\Psi_{B}\right|^{2},
\end{aligned}
$$

where $g_{B F}=2 \pi \hbar^{2} a_{B F} / m_{R}$ with the boson-fermion reduced mass $m_{R}=m_{B} m_{F} /\left(m_{B}+m_{F}\right)$, where $a_{B F}$ is the boson-fermion scattering length.

The terms in the first round bracket on the right-hand side of Eq. (2.3) are the standard Gross-Pitaevskii terms for the bosons and are related to a Schrödinger-like equation [13]. However, terms in the second round bracket are derived from the hydrodynamic equation of motion of the fermions [11]. Hence, the second kinetic energy term has a different mass factor $6 m_{F}$ and not the conventional Schrödinger mass factor $2 m_{B}$ as in the first integral. Finally, the last term in this equation corresponds to an interaction between bosons and fermions. The interaction between fermions in spin polarized state is highly suppressed due to Pauli blocking and has been neglected in Eq. (2.3) and will be neglected throughout this paper.

Recently, Jezek et al. [18] used the Thomas-FermiWeizsäcker kinetic energy term $T_{F}$ of fermions in their formulation which, in our notation, will correspond to a fermionic kinetic energy of $\hbar^{2}\left|\nabla_{\mathbf{r}} \sqrt{n_{F}}\right|^{2} /\left(9 m_{F}\right)$ in Eq. (2.3) in place of the present term $\hbar^{2}\left|\boldsymbol{\nabla}_{\mathbf{r}} \sqrt{n_{F}}\right|^{2} /\left(6 m_{F}\right)$. This kinetic energy term contributes little to this problem compared to the dominating $3 A\left|n_{F}\right|^{5 / 3} / 5$ term in Eq. (2.3) and is usually neglected in the Thomas-Fermi approximation. However, its inclusion leads to an analytic solution for the probability density everywhere [18]. For a discussion of these two fermionic kinetic energy terms we refer the reader to Refs. $[11,18,19]$.

With the Lagrangian density (2.3), the Euler-Lagrange equations of motion become [12]

$$
\begin{gathered}
{\left[-i \hbar \frac{\partial}{\partial t}-\frac{\hbar^{2} \boldsymbol{\nabla}_{\mathbf{r}}^{2}}{2 m_{B}}+V_{B}(\mathbf{r})+g_{B B} n_{B}+g_{B F} n_{F}\right] \Psi_{B}(\mathbf{r}, t)=0} \\
{\left[-i \hbar \frac{\partial}{\partial t}-\frac{\hbar^{2} \nabla_{\mathbf{r}}^{2}}{6 m_{F}}+V_{F}(\mathbf{r})+A\left|n_{F}\right|^{2 / 3}+g_{B F} n_{B}\right] \sqrt{n_{F}(\mathbf{r}, t)}=0 .}
\end{gathered}
$$

When the nonlinearity in Eq. (2.5) is very large, the kinetic energy term in this equation can be neglected and the time-independent stationary form of this equation becomes

$$
n_{F}=\frac{\left[\max \left(0,\left\{\epsilon_{F}-V_{F}(\mathbf{r})-g_{B F} n_{B}\right\}\right)\right]^{3 / 2}}{A^{3 / 2}},
$$

which is the generalization of Eq. (2.2) in the presence of boson-fermion coupling. Equation (2.6) has been used by Modugno et al. [10] for an analysis of a DFG-BEC mixture. In actual experimental condition the nonlinearity in Eq. (2.5) 
is quite large and Eq. (2.6) is a good approximation.

For the study of bright solitons we shall reduce Eqs. (2.4) and (2.5) to the minimal one-dimensional form under the action of stronger radial trapping. The one-dimensional form is appropriate for studying bright solitons in the so-called cigar-shaped quasi-one-dimensional geometry where $\nu \ll 1$. For radially-bound and axially-free solitons we eventually set $\nu=0$. In this case the dynamical equations can be reduced to strict one-dimensional coupled NLS equations without any trap. We perform this reduction below where we take $V_{B}(\mathbf{r})$ $=V_{F}(\mathbf{r})=\frac{1}{2} m_{B} \omega^{2}\left(\rho^{2}+\nu^{2} z^{2}\right)$ which corresponds to a reduction of $\omega_{F}$ and $\nu \omega_{F}$ in $V_{F}(\mathbf{r})$ by a factor $\sqrt{m_{B} / m_{F}}$ as in the study by Modugno et al. [10] and Jezek et al. [18].

For $\nu=0$, Eqs. (2.4) and (2.5) can be reduced to an effective one-dimensional form by considering solutions of the type $\Psi_{B}(\mathbf{r}, t)=\phi_{B}(z, t) \psi_{B}^{(0)}(\rho)$ and $\sqrt{n_{F}(\mathbf{r}, t)}=\phi_{F}(z, t) \psi_{F}^{(0)}(\rho)$, where

$$
\left|\psi_{i}^{(0)}(\rho)\right|^{2} \equiv \frac{M_{i} \omega}{\pi \hbar} \exp \left(-\frac{M_{i} \omega \rho^{2}}{\hbar}\right)
$$

and $i=B, F$ represents bosons and fermions and $M_{B}=m_{B}$ and $M_{F}=\sqrt{3 m_{B} m_{F}}$. The expression (2.7) corresponds to the respective ground-state wave function in the absence of nonlinear interactions and satisfies

$$
\begin{gathered}
-\frac{\hbar^{2}}{2 m_{B}} \nabla_{\rho}^{2} \psi_{B}^{(0)}+\frac{1}{2} m_{B} \omega^{2} \rho^{2} \psi_{B}^{(0)}=\hbar \omega \psi_{B}^{(0)}, \\
-\frac{\hbar^{2}}{6 m_{F}} \nabla_{\rho}^{2} \psi_{F}^{(0)}+\frac{1}{2} m_{B} \omega^{2} \rho^{2} \psi_{F}^{(0)}=\sqrt{\frac{m_{B}}{3 m_{F}}} \hbar \omega \psi_{F}^{(0)},
\end{gathered}
$$

with normalization $2 \pi \int_{0}^{\infty}\left|\psi_{i}^{(0)}(\rho)\right|^{2} \rho d \rho=1$. Now the dynamics is carried by $\phi_{i}(z, t)$ and the radial dependence is frozen in the ground state $\psi_{i}^{(0)}(\rho)$. The factorization of $\Psi_{B}$ and $\sqrt{n_{F}}$ above follows from the structure of the mathematical equations (2.4) and (2.5). Although $n_{F}$ gives the probability density of DFG it may not be to the point to associate $\psi_{F}^{0}$ and $\phi_{F}$ to physical fermionic one-particle wave functions. The true fermionic wave function has the form of a many-particle Slater determinant. Nevertheless, the functions $\psi_{F}^{0}$ and $\phi_{F}$ could be regarded as mathematical functions related to fermionic density [8]. In the quasi-one-dimensional cigarshaped geometry the linear fermionic and bosonic probability densities are given by $\left|\phi_{F}(z, t)\right|^{2}$ and $\left|\phi_{B}(z, t)\right|^{2}$, respectively.

Averaging over the radial mode $\psi_{i}^{(0)}(\rho)$, i.e., multiplying Eqs. (2.4) and (2.5) by $\psi_{i}^{(0) *}(\rho)$ and integrating over $\rho$, we obtain the following one-dimensional dynamical equations [20]:

$$
\left[-i \hbar \frac{\partial}{\partial t}-\frac{\hbar^{2}}{2 m_{B}} \frac{\partial^{2}}{\partial z^{2}}+F_{B B}\left|\phi_{B}\right|^{2}+F_{B F}\left|\phi_{F}\right|^{2}\right] \phi_{B}(z, t)=0,
$$

$$
\left[-i \hbar \frac{\partial}{\partial t}-\frac{\hbar^{2}}{6 m_{F}} \frac{\partial^{2}}{\partial z^{2}}+F_{F F}\left|\phi_{F}\right|^{4 / 3}+F_{B F}\left|\phi_{B}\right|^{2}\right] \phi_{F}(z, t)=0,
$$

where

$$
\begin{gathered}
F_{B B}=g_{B B} \frac{\int_{0}^{\infty}\left|\psi_{B}^{(0)}\right|^{4} \rho d \rho}{\int_{0}^{\infty}\left|\psi_{B}^{(0)}\right|^{2} \rho d \rho}=g_{B B} \frac{m_{B} \omega}{2 \pi \hbar}, \\
F_{B F}=g_{B F} \frac{\int_{0}^{\infty}\left|\psi_{F}^{(0)}\right|^{2}\left|\psi_{B}^{(0)}\right|^{2} \rho d \rho}{\int_{0}^{\infty}\left|\psi_{B}^{(0)}\right|^{2} \rho d \rho}=g_{B F} \frac{M_{B F} \omega}{\pi \hbar}, \\
F_{F F}=A \frac{\int_{0}^{\infty}\left|\psi_{F}^{(0)}\right|^{2+4 / 3} \rho d \rho}{\int_{0}^{\infty}\left|\psi_{B}^{(0)}\right|^{2} \rho d \rho}=\frac{3 A}{5}\left[\frac{M_{F} \omega}{\pi \hbar}\right]^{2 / 3} .
\end{gathered}
$$

In Eq. (2.13) $M_{B F}=M_{B} M_{F} /\left(M_{B}+M_{F}\right)$. In Eqs. (2.10) and (2.11) the normalization is given by $\int_{-\infty}^{\infty}\left|\phi_{i}(z, t)\right|^{2} d z=N_{i}$. In these equations we have set the anisotropy parameter $\nu=0$ to remove the axial trap and thus to generate axially free quasione-dimensional solitons.

For calculational purposes it is convenient to reduce the set of Eqs. (2.10) and (2.11) to dimensionless form by introducing convenient dimensionless variables. Although the algebra is quite straightforward, the expressions become messy with different factors of masses. As we shall not be interested in a particular boson-fermion system in this paper, but will be concerned with the formation of fermionic bright solitons in general, we take in the rest of this paper $m_{B}=3 m_{F}$ $=m\left({ }^{87} \mathrm{Rb}\right)$, where $m\left({ }^{87} \mathrm{Rb}\right)$ is the mass of ${ }^{87} \mathrm{Rb}$, and whence $m_{R}=3 m_{F} / 4, M_{B}=M_{F}=m_{B}$, and $M_{B F}=m_{B} / 2$. In the two experimental situations of Refs. [4,5] $m_{B} \approx 3 m_{F}$.

In Eqs. (2.10) and (2.11), we consider the dimensionless variables $\tau=t \omega / 2, y=z / l, \chi_{i}=\sqrt{\left(l / N_{i}\right)} \phi_{i}$, with $l=\sqrt{\hbar /\left(\omega m_{B}\right)}$, so that

$$
\begin{aligned}
& {\left[-i \frac{\partial}{\partial \tau}-\frac{d^{2}}{d y^{2}}+N_{B B}\left|\chi_{B}\right|^{2}+N_{B F}\left|\chi_{F}\right|^{2}\right] \chi_{B}(y, \tau)=0,} \\
& {\left[-i \frac{\partial}{\partial \tau}-\frac{d^{2}}{d y^{2}}+N_{F B}\left|\chi_{B}\right|^{2}+N_{F F}\left|\chi_{F}\right|^{4 / 3}\right] \chi_{F}(y, \tau)=0,}
\end{aligned}
$$

where $N_{B B}=4 a_{B B} N_{B} / l, N_{B F}=8 a_{B F} N_{F} / l, N_{F B}=8 a_{B F} N_{B} / l$, and $N_{F F}=9\left(6 \pi N_{F}\right)^{2 / 3} / 5$. In Eqs. (2.15) and (2.16), the normalization condition is given by $\int_{-\infty}^{\infty}\left|\chi_{i}(y, \tau)\right|^{2} d y=1$. Equations (2.15) and (2.16) are the coupled one-dimensional NLS equations describing the formation of solitons in the 
DFG-BEC mixture in a cigar-shaped quasi-one-dimensional geometry.

In Eqs. (2.15) and (2.16) the term $N_{F F}\left|\chi_{F}\right|^{4 / 3}$ represents a very strong Pauli repulsion which increases with the fermion number $N_{F}$. The purpose of this study is to show that a sufficiently strong attractive boson-fermion coupling term $N_{F B}\left|\chi_{B}\right|^{2}$ can overcome this repulsion and form the bright solitons.

\section{NUMERICAL RESULT}

We solve the coupled mean-field-hydrodynamic equations (2.15) and (2.16) for bright solitons numerically using a time-iteration method based on the Crank-Nicholson discretization scheme elaborated in Ref. [21]. We discretize the mean-field-hydrodynamic equation using time step 0.0005 and space step 0.025 .

We performed the time evolution of the set of equations (2.15) and (2.16) introducing harmonic oscillator potentials $y^{2}$ in these equations and setting the nonlinear terms to zero: $N_{B B}=N_{B F}=N_{F B}=N_{F F}=0$ and starting with the eigenfunctions of the linear harmonic oscillator problem, e.g., with $\chi_{B}(y, \tau)=\chi_{F}(y, \tau)=\pi^{-1 / 4} \exp \left(-y^{2} / 2\right) \exp (-i \tau)$. The introduction of the extra harmonic oscillator potential in these equations only aids in starting the time evolution with an exact analytic solution. In the end the harmonic oscillator potentials will be set equal to zero and will have no effect on the final wave function for solitons. During the course of time evolution the nonlinear terms are switched on very slowly and the resultant solutions iterated until convergence was obtained. Then the time evolution is continued and the harmonic oscillator potential terms in both bosonic and fermionic equations are slowly switched off by reducing the $y^{2}$ term to zero in 10000 steps of time evolution. Then the resultant solutions are iterated about 50000 times for convergence without any harmonic oscillator potential. If converged solutions are obtained, they correspond to the required axially free bright solutions in the absence of any axial potential. In our numerical investigation as in the theoretical study of Refs. $[12,18]$ we use $\omega=2 \pi \times 100 \mathrm{~Hz}$, and take $m_{B}$ as the mass of ${ }^{87} \mathrm{Rb}$. Consequently, the unit of length $l \approx 1 \mu \mathrm{m}$ and unit of time $2 / \omega \approx 3 \mathrm{~ms}$.

First we solve Eqs. (2.15) and (2.16) with $N_{F}=N_{B}=1000$, $a_{B B}=5 \mathrm{~nm}$ and $a_{B F}=-20 \mathrm{~nm}$. This value of $a_{B B}$ is the experimental scattering length of $\mathrm{Rb}$ atoms [13], and $a_{B F}=$ $-20 \mathrm{~nm}$ is the experimental scattering length of the Rb-K system $[5,6]$. With these parameters the nonlinearities in Eqs. (2.15) and (2.16) are $N_{B B}=20, N_{B F}=-160, N_{F B}=-160$, and $N_{F F}=274.6$. The converged bright solitons are plotted in Fig. 1 . In this case the fermionic and bosonic functions $\phi_{F}$ and $\phi_{B}$, respectively, have similar spatial extentions. It is possible to have solitons with different extensions in space by varying the parameters of the system. We took the experimental values for the scattering lengths in Fig. 1. However, the scattering length can be manipulated in the boson-fermion ${ }^{6} \mathrm{Li}^{23}{ }^{23} \mathrm{Na}$ and ${ }^{40} \mathrm{~K}-{ }^{87} \mathrm{Rb}$ systems near the recently discovered Feshbach resonances in them [22] by varying a background magnetic field. Thus by varying the scattering length and the number of atoms we could arrive at different values of nonlinearity than in Fig. 1.

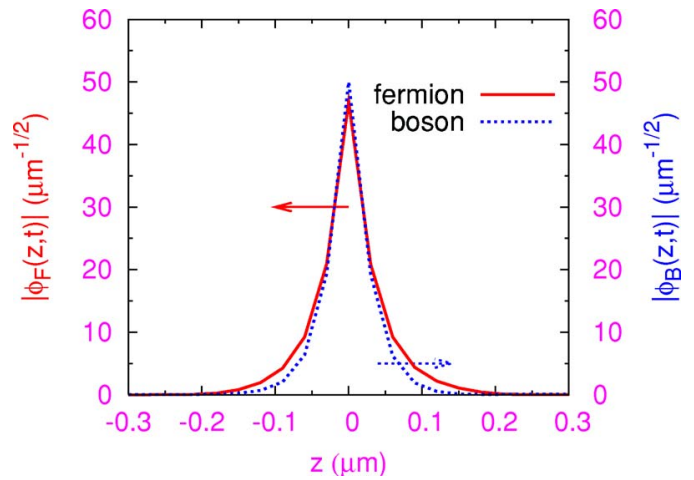

FIG. 1. (Color online) The stationary function $\left|\phi_{i}(z, t)\right|$ for axially-free bosonic (dotted line) and fermionic (full line) bright solitons vs $z$ for $N_{F}=N_{B}=1000, a_{B B}=5 \mathrm{~nm}, a_{B F}=-20 \mathrm{~nm}$, harmonic oscillator length $l \approx 1 \mu \mathrm{m}$ and $\nu=0$. The arrows in dotted and full lines indicate the bosonic and fermionic axes, respectively. The nonlinearity parameters are $N_{B B}=20, N_{B F}=-160, N_{F B}=-160$, and $N_{F F}=274.6$.

To simulate a different situation of nonlinearity parameters next we take $a_{B B}=-1 \mathrm{~nm}, a_{B F}=-1.875 \mathrm{~nm}, N_{F}=1000$, and $N_{B}=10000$. So that $N_{B B}=-40, N_{B F}=-15, N_{F B}=-150$, and $N_{F F}=274.6$. In this case the profiles of the bright solitons shown in Fig. 2 are very different from those in Fig. 1. In Fig. 1 both the solitons are localized to a small region in space, whereas in Fig. 2 only the bosonic soliton is localized to a small region in space whereas the fermionic soliton extends to a very large region of space.

Next we consider $N_{F}=1000, N_{B}=10000, a_{B B}=0.5 \mathrm{~nm}$, and $a_{B F}=-3.75 \mathrm{~nm}$, so that the nonlinearity parameters are $N_{B B}=20, N_{B F}=-30, N_{F B}=-300$, and $N_{F F}=274.6$. The profiles of the solitons in Fig. 3 are different from those in Figs. 1 and 2. In this case the bosonic function extends over a longer region in space than the fermionic function. In Figs. 1 and 2 it was the fermionic function that extends over a longer region in space.

In Fig. 2 the nonlinearity $N_{F B}$ appearing in the fermion equation is less attractive compared to that in Figs. 1 and 3.

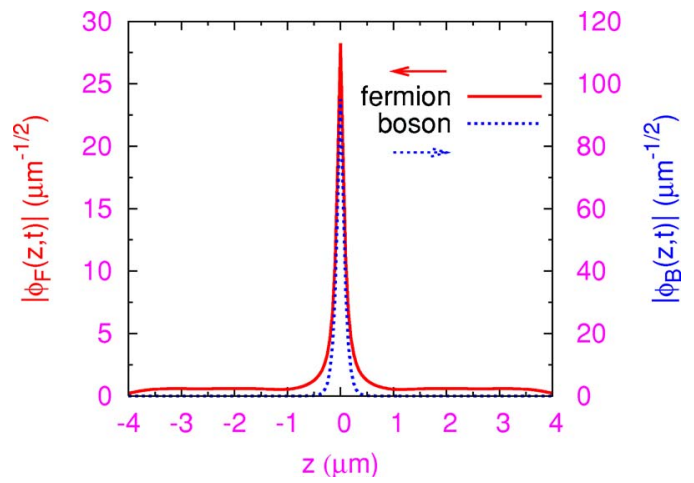

FIG. 2. (Color online) The stationary function $\left|\phi_{i}(z, t)\right|$ for axially free bosonic (dotted line) and fermionic (solid line) bright solitons vs $z$ for $N_{F}=1000, N_{B}=10000, a_{B B}=-1 \mathrm{~nm}, a_{B F}=-1.875 \mathrm{~nm}$, harmonic oscillator length $l \approx 1 \mu \mathrm{m}$ and $\nu=0$. The arrows in dotted and full lines indicate the bosonic and fermionic axes, respectively. The nonlinearity parameters are $N_{B B}=-40, N_{B F}=-15, N_{F B}=-150$, and $N_{F F}=274.6$. 


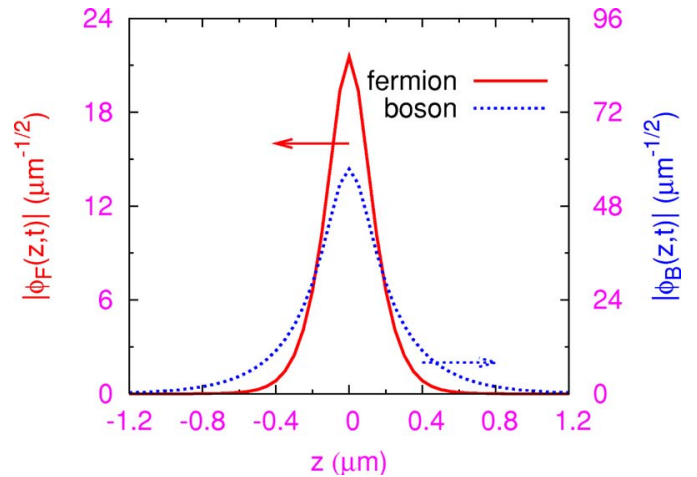

FIG. 3. (Color online) The stationary function $\left|\phi_{i}(z, t)\right|$ for axially free bosonic (dotted line) and fermionic (solid line) bright solitons vs $z$ for $N_{F}=1000, N_{B}=10000, a_{B B}=0.5 \mathrm{~nm}, a_{B F}=-3.75 \mathrm{~nm}$, $\nu=0$ and harmonic oscillator length $l \approx 1 \mu \mathrm{m}$. The arrows in dotted and full lines indicate the bosonic and fermionic axes, respectively. The nonlinearity parameters are $N_{B B}=20, N_{B F}=-30, N_{F B}=-300$, and $N_{F F}=274.6$.

Hence the resultant nonlinear interaction in the fermion component is less attractive and hence the fermionic soliton extends to a large distance in space. If the attraction in $N_{F B}$ is further reduced the fermionic soliton ceases to bind.

Hence by manipulating the parameters one could have different situations of localization of the solitons. One could either have both the solitons extending up to similar distance in space as in Fig. 1 or one of the solitons extending over a longer region in space as in Figs. 2 and 3. It is worth emphasizing that in the fermionic equation (2.16) the diagonal nonlinearity $N_{F F}$ is repulsive, hence the binding solely comes from the attractive off-diagonal nonlinearity $N_{F B}$ corresponding to an attractive boson-fermion interaction. Hence for a fermionic soliton to appear $N_{F B}$ and $a_{B F}$ are always taken to be negative or attractive. Consequently, $N_{B F}$ is also negative. Finally, terms $N_{B B}$ and $a_{B B}$ could be either positive or negative. When these terms are positive or repulsive the bosonic solitons are formed due to an attractive or negative $N_{B F}$, as in Fig. 3.

Next we study the stability of the bright solitons numerically. We consider the soliton of Fig. 3 and during time evolution we suddenly jump at $t=100 \mathrm{~ms}$ the nonlinearity $N_{B F}$ from -30 to -33 and the nonlinearity $N_{F B}$ from -300 to -330 . This can be achieved by manipulating a background magnetic field near a Feshbach resonance [22] in the bosonfermion interaction and thus varying the boson-fermion scattering length by $10 \%$. Due to the sudden change in the nonlinearity the bosonic and fermionic bright solitons are set into stable breathing oscillation. The evolution of the wave function profile of the two solitons are shown in Fig. 4. The solitons are found to execute stable non-periodic breathing oscillation. In Fig. 5 we plot the root-mean-square size $\langle z\rangle_{\text {r.m.s. }}$ of the bosonic and fermionic solitons of Fig. 4 as a function of time. The breathing oscillation of the two solitons after the perturbation is applied results in the stable nonperiodic oscillation of the root-mean-square sizes illustrated in Fig. 5. As, after applying the perturbation, the boson-fermion attraction has been increased this corresponds to a reduction in the root-mean-square size $\langle z\rangle_{\text {r.m.s. }}$ as we find in Fig. 5. The
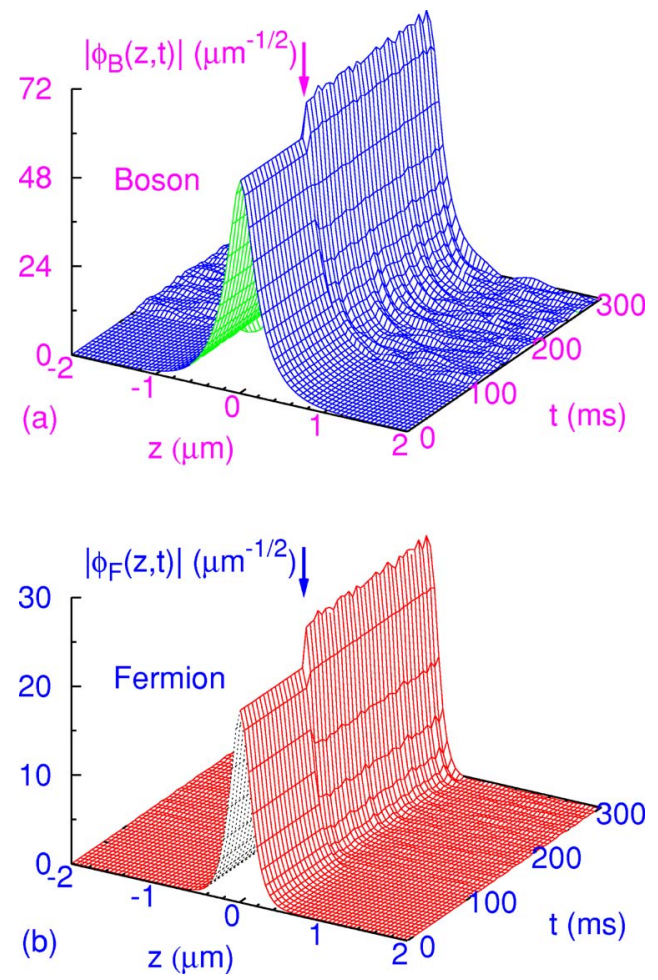

FIG. 4. (Color online) The function $\left|\phi_{i}(z, t)\right|$ for (a) bosonic and (b) fermionic bright solitons vs $z$ and $t$ for the solitons of Fig. 3. At $t=0 N_{F}=1000, N_{B}=10000, a_{B B}=0.5 \mathrm{~nm}, a_{B F}=-3.75 \mathrm{~nm}, \nu=0$ and harmonic oscillator length $l \approx 1 \mu \mathrm{m}$. The nonlinearity parameters at $t=0$ are $N_{B B}=20, N_{B F}=-30, N_{F B}=-300$, and $N_{F F}=274.6$. At $t$ $=100 \mathrm{~ms}$ (marked by arrows) the bright solitons are set into small breathing oscillations by suddenly jumping the nonlinearities $N_{B F}$ and $N_{F B}$ to -33 and -330 , respectively.

steady propagation of the solitons in Fig. 4 and the stable oscillation of their root-mean-square sizes in Fig. 5 after the perturbation is applied will demonstrate the stability of the solitons.

Finally, we consider the fermionic bright solitons formed on a periodic optical-lattice potential. For that purpose we include in Eqs. (2.15) and (2.16) the following optical-lattice potential formed by a standing-wave laser beam [17]:

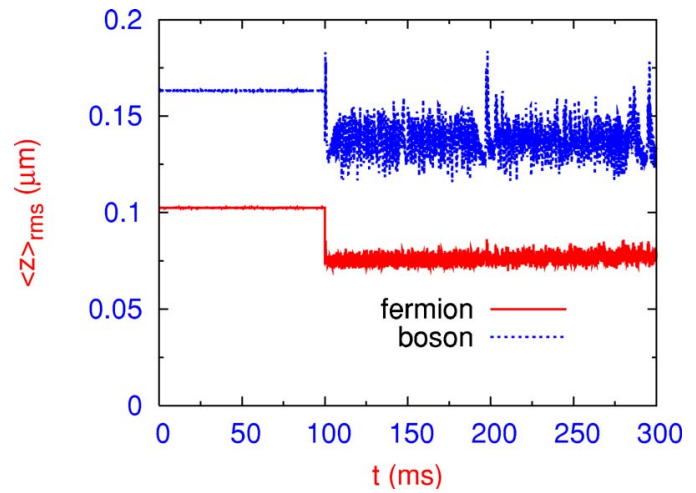

FIG. 5. (Color online) The root-mean-square size $\langle z\rangle_{\text {r.m.s. }}$ of the bosonic (dotted line) and fermionic (full line) solitons of Fig. 4 vs time. 


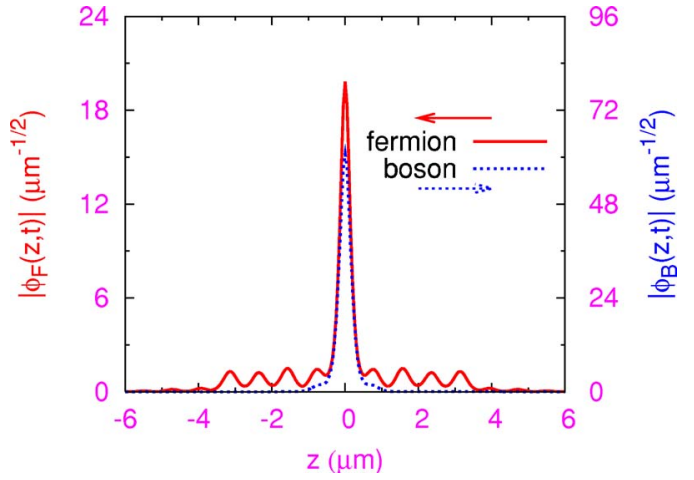

FIG. 6. (Color online) The stationary function $\left|\phi_{i}(z, t)\right|$ for bosonic (dotted line) and fermionic (full line) bright solitons vs $z$ for $N_{F}=1000, N_{B}=10000, a_{B B}=0.3 \mathrm{~nm}, a_{B F}=-2.375 \mathrm{~nm}, \nu=0$ and harmonic oscillator length $l \approx 1 \mu \mathrm{m}$ in the presence of the opticallattice potential $V(y)=100 \sin ^{2}(4 y)$. The arrows in dotted and full lines indicate the bosonic and fermionic axes, respectively. The nonlinearity parameters are $N_{B B}=12, N_{B F}=-19, N_{F B}=-190$, and $N_{F F}=274.6$.

$$
V_{\mathrm{OP}}=V_{0} \sin ^{2}(2 \pi y / \lambda),
$$

where $V_{0}$ is the strength, and $\lambda$ is the wave length of the laser. In our calculation we take $V_{0}=100$ and $\lambda=\pi / 2$. To solve Eqs. (2.15) and (2.16) with this optical-lattice potential and desired nonlinearities, we again start the time evolution with the solution of the linear oscillator problem. In the course of time evolution the nonlinearities and the opticallattice potential are slowly introduced and eventually the harmonic oscillator potential is slowly removed. Then the final solutions are iterated for convergence. The resultant soliton wave functions are plotted in Fig. 6 for $N_{F}=1000, N_{B}$ $=10000, a_{B B}=0.3 \mathrm{~nm}$, and $a_{B F}=-2.375 \mathrm{~nm}$. The opticallattice potential introduces modulations in the solitonic wave function. For the parameters of Fig. 6 the modulations are more prominent on the fermionic soliton than the bosonic one. By changing the parameters it is possible to have modulations on the bosonic soliton as well.

\section{SUMMARY}

We use a coupled set of time-dependent mean-fieldhydrodynamic equations for a boson-fermion mixture to study the formation of fermionic bright soliton in a DFG as a stationary state. In this study we take the boson-boson interaction to be both attractive and repulsive and the bosonfermion interaction to be attractive. An attractive bosonfermion interaction is necessary for the formation of a fermionic bright soliton as the diagonal nonlinearity $N_{F F}$ in the fermion-fermion system is always repulsive.

In the present study we demonstrate that stable solitons can be formed in coupled NLS equations for the bosonfermion mixture with repulsive diagonal nonlinearities and attractive off-diagonal nonlinearities above some cutoff values. In another study [23] we showed the possibility of the formation of bright solitons in coupled bosonic condensates with intraspecies repulsion supported by interspecies attraction. The stability of the present fermionic and bosonic solitons is demonstrated through their sustained breathing oscillation initiated by a sudden jump in the boson-fermion scattering length. Bright solitons have been created experimentally in attractive BECs in three dimensions in the presence of radial trapping only without any axial trapping [15]. In view of this, fermionic bright solitons can be observed in the laboratory in the presence of radial trapping only in a mixture of a DFG and BEC. We also suggest the possibility of the formation of fermionic solitons on a periodic opticallattice potential. In the present investigation we used a set of mean-field equations for the DFG-BEC mixture. A proper treatment of the DFG should be performed using a fully antisymmetrized many-body Slater determinant wave function [8], as in the case of atomic and molecular scattering involving many electrons [24]. However, in view of the success of the hydrodynamic model in other contexts [12,25], we do not believe that the present conclusion about the existence of robust fermionic solitons in a DFG-BEC mixture to be so peculiar as to have no general validity.

Note added in proofs: Recently, we became aware of reaching a similar conclusion from a numerical solution of a model using an antisymmetrized wave function for the fermion wave function [26]. We apologize for missing this article before.

\section{ACKNOWLEDGMENTS}

The work is supported in part by the CNPq of Brazil.
[1] B. DeMarco and D. S. Jin, Science 285, 1703 (1999).

[2] K. M. O'Hara, S. L. Hemmer, M. E. Gehm, S. R. Granade, and J. E. Thomas, Science 298, 2179 (2002).

[3] F. Schreck, L. Khaykovich, K. L. Corwin, G. Ferrari, T. Bourdel, J. Cubizolles, and C. Salomon, Phys. Rev. Lett. 87, 080403 (2001); A. G. Truscott, K. E. Strecker, W. I. McAlexander, G. B. Partridge, and R. G. Hulet, Science 291, 2570 (2001).

[4] Z. Hadzibabic, C. A. Stan, K. Dieckmann, S. Gupta, M. W. Zwierlein, A. Gorlitz, and W. Ketterle, Phys. Rev. Lett. 88, 160401 (2002).
[5] G. Modugno, G. Roati, F. Riboli, F. Ferlaino, R. J. Brecha, and M. Inguscio, Science 297, 2240 (2002).

[6] G. Roati, F. Riboli, G. Modugno, and M. Inguscio, Phys. Rev. Lett. 89, 150403 (2002).

[7] K. E. Strecker, G. B. Partridge, and R. G. Hulet, Phys. Rev. Lett. 91, 080406 (2003); Z. Hadzibabic, S. Gupta, C. A. Stan, C. H. Schunck, M. W. Zwierlein, K. Dieckmann, and W. Ketterle, ibid. 91, 160401 (2003).

[8] K. Molmer, Phys. Rev. Lett. 80, 1804 (1998).

[9] R. Roth, Phys. Rev. A 66, 013614 (2002); R. Roth, and H. Feldmeier, ibid. 65, 021603(R) (2002); T. Miyakawa, T. Su- 
zuki, and H. Yabu, ibid. 64, 033611 (2001); X.-J. Liu, M. Modugno, and H. Hu, ibid. 68, 053605 (2003); X.-J. Liu and H. Hu, ibid. 67, 023613 (2003); L. Vichi, M. Amoruso, A. Minguzzi, S. Stringari, and M. Tosi, Eur. Phys. J. D 11, 335 (2000).

[10] M. Modugno, F. Ferlaino, F. Riboli, G. Roati, G. Modugno, and M. Inguscio, Phys. Rev. A 68, 043626 (2003).

[11] P. Capuzzi, A. Minguzzi, and M. P. Tosi, Phys. Rev. A 69, 053615 (2004); 68, 033605 (2003); 67, 053605 (2003).

[12] S. K. Adhikari, Phys. Rev. A 70, 043617 (2004).

[13] F. Dalfovo, S. Giorgini, L. P. Pitaevskii, and S. Stringari, Rev. Mod. Phys. 71, 463 (1999); L. P. Pitaevskii and S. Stringari, Bose-Einstein Condensation (Clarendon Press, Oxford, 2003).

[14] Y. S. Kivshar and G. P. Agrawal, Optical Solitons-From Fibers to Photonic Crystals (Academic Press, San Diego, 2003); A. Hasegawa and Y. Kodama, Solitons in Optical Communications (Clarendon Press, Oxford, 1995); G. P. Agrawal, Nonlinear Fiber Optics, Second Edition (Academic Press, San Diego, 1995).

[15] K. E. Strecker, G. B. Partridge, A. G. Truscott, and R. G. Hulet, Nature (London) 417, 150 (2002); L. Khaykovich, F. Schreck, G. Ferrari, T. Bourdel, J. Cubizolles, L. D. Carr, Y. Castin, and C. Salomon, Science 296, 1290 (2002).

[16] V. M. Perez-Garcia, H. Michinel, and H. Herrero, Phys. Rev. A 57, 3837 (1998); U. Al Khawaja, H. T. C. Stoof, R. G. Hulet, K. E. Strecker, and G. B. Partridge, Phys. Rev. Lett. 89, 200404 (2002); S. K. Adhikari, New J. Phys. 5, 137 (2003).
[17] F. S. Cataliotti, S. Burger, C. Fort, P. Maddaloni, F. Minardi, A. Trombettoni, A. Smerzi, and M. Inguscio, Science 293, 843 (2001); M. Greiner, O. Mandel, T. Esslinger, T. W. Hänsch, and I. Bloch, Nature (London) 415, 39 (2002); O. Morsch, J. H. Muller, M. Cristiani, D. Ciampini, and E. Arimondo, Phys. Rev. Lett. 87, 140402 (2001); S. K. Adhikari and P. Muruganandam, Phys. Lett. A 310, 229 (2003); S. K. Adhikari, Eur. Phys. J. D 25, 161 (2003); Phys. Rev. A 72, 013619 (2005).

[18] D. M. Jezek, M. Barranco, M. Guilleumas, R. Mayol, and M. Pi, Phys. Rev. A 70, 043630 (2004).

[19] M. Pi, X. Viñas, F. Garcias, and M. Barranco, Phys. Lett. B 215, 5 (1988).

[20] F. K. Abdullaev and R. Galimzyanov, J. Phys. B 36, 1099 (2003).

[21] S. K. Adhikari and P. Muruganandam, J. Phys. B 35, 2831 (2002); P. Muruganandam and S. K. Adhikari, ibid. 36, 2501 (2003).

[22] C. A. Stan, M. W. Zwierlein, C. H. Schunck, S. M. F. Raupach, and W. Ketterle, Phys. Rev. Lett. 93, 143001 (2004); S. Inouye, J. Goldwin, M. L. Olsen, C. Ticknor, J. L. Bohn, and D. S. Jin, ibid. 93, 183201 (2004).

[23] S. K. Adhikari, Phys. Lett. A 346, 179 (2005); Phys. Rev. A 63, 043611 (2001).

[24] P. K. Biswas and S. K. Adhikari, J. Phys. B 33, 1575 (2000); 31, L737 (1998); 31, 3147 (1998); 31, L315 (1998).

[25] S. K. Adhikari, J. Phys. B 38, 3607 (2005).

[26] T. Karpiuk et al., Phys. Rev. Lett. 93, 100401 (2004). 